\title{
Neural Network Based System Identification for Autonomous Flight of an Eagle Helicopter
}

\author{
Mahendra Kumar Samal, Sreenatha Anavatti, \\ Matthew Garratt \\ School of Aerospace, Civil and Mechanical Engg. University of New \\ South Wales at Australian Defence Force Academy, Canberra, \\ ACT-2600, Australia (e-mail: \{m.samal, agsrenat, \\ m.garratt\}@adfa.edu.au).
}

\begin{abstract}
Neural Network Identification (NNID) for modeling the dynamics of a miniature Eagle helicopter is presented in this paper. Off-line and on-line identification is carried out for both coupled and decoupled dynamics of the helicopter from the flight test data. For both the cases, identification results and the error statistics are provided. The off-line identification performs better due to sufficient training time and data. Results indicate neural network based black-box method is suitable for modeling the nonlinear dynamics of the helicopter. This can be further applied for the design of Automatic Flight Control System (AFCS).
\end{abstract}

Keywords: System Identification, Artificial Neural network, Autonomous Vehicles, Dynamic modeling, Helicopter Dynamics.

\section{INTRODUCTION}

Rotary wing Unmanned Aerial Vehicles (UAVs) have been an active area of research in the last decade due to their attractive features and capabilities. These include vertical take-off and landing from unimproved terrain and flight deck, hovering and different complicated maneuvers in urban environments that may not be possible with conventional fixed wing UAVs. Rotary-wing UAVs can be employed for various military and civilian applications such as real-time reconnaissance, surveillance of enemy locations, search and rescue missions, weather data collection, bush fire monitoring, inspection of bridges and power lines, agricultural crop dusting and different airborne operations.

Two autonomous helicopter platforms are under development at UNSW@ADFA with an objective to develop Flight Control System (FCS) for fully autonomous flight. These are the Japanese built RMAX helicopter and Hirobo "Eagle" helicopter. These platforms have been instrumented with different sensor and avionics for measurements, processing and control.

The complex, asymmetric and nonlinear dynamics make the control of helicopter extremely challenging. To design a suitable flight control system, a good mathematical model of the helicopter is required. This can be obtained by suitable identification technique using the flight data. Besides this, the flight vehicle (helicopter) system identification can also be used for model validation, in-flight simulators, simulator data base, and for expanding the flight test envelope.

Various system identification techniques that can be broadly classified as parametric and non parametric techniques have been successfully applied for flight vehicle system identification. These system identification methods can also be classified as conventional and non conventional methods. The parametric approach requires the mathematical model, order and structure of the system to be known a priori. In the parametric approach, conventional methods such as Maximum Likelihood Estimation method (MLE), Modified Maximum Likelihood Estimation method (MMLE) and Kalman Filtering (KF) [Mettler et al. (2002); Jategaonkar et al. (2004); Chowdhary and Jategaonkar (2006); Kallapur et al. (2006)] have been employed to model the flight dynamics. In this approach, the stability and control parameters of the flight vehicle are estimated and the computed parameters are then used in the assumed aerodynamic model to model the dynamics. Some research using the non conventional methods like the neural network has also been applied in the parametric approach [Raol (1994); Raisinghani and Ghosh (1998); Ghosh et al. (1998)].

Sometimes, it may not be possible to know the exact order and structure of the mathematical model to apply the parametric estimation techniques. Due to the complex, coupled and nonlinear dynamics of the helicopter, a higher order model description is needed to completely describe the system. In addition, the inherent measurement noise from sensors and high level of vibration leads to further complexity. Due to these problems, the alternate nonparametric approach using non-conventional methods such as Fuzzy logic and neural network have recently gained popularity for flight vehicle system identification. The Neural network based black-box system identification technique is used by Aydogan Savran and Becerikli (2006) and Kumar et al. (2006) for flight vehicle system identifications using flight data for piloted aircraft. 
In this paper, the neural network is applied to model the coupled nonlinear dynamics of a rotary wing UAV. The flight data from the miniature Eagle helicopter is utilized for both off-line and on-line identification. Numerical results as well as statistical analysis are carried out to validate the proposed technique.

Section 2 details the platform used for the experimentation and data collection while section 3 deals with the formulation for system identification and neural network technique. Results and discussion are provided in section 4 and section 5 concludes the paper.

\section{PLATFORM DESCRIPTION}

In this section a brief overview of the platform and sensors used for flight experiments is provided. The platform used for experiments (Figure 1) is a Hirobo "Eagle" 60 size radio controlled helicopter, modified to use a brushless DC motor in lieu of the standard internal combustion engine. The design incorporates a single main rotor and a single tail-rotor for anti-torque compensation. Five servo actuators: collective, throttle, aileron, elevator and tail rotor pitch are used to control the helicopter.

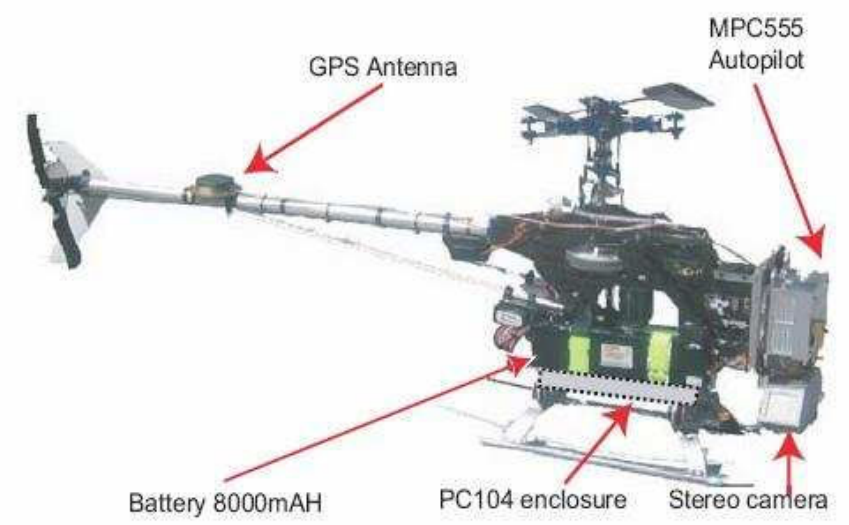

Fig. 1. The miniature Eagle helicopter platform

\subsection{Autopilot Systems}

The autopilot system consists of the MPC555 based autopilot and PC104 based flight computer (Figure 2).

MPC555 based Autopilot: This unit is made in-house based on a Motorola MPC555 microcontroller. The autopilot is interfaced to an Inertial Measurement Unit (IMU) comprising accelerometers, gyroscopes and magnetometers which enable the autopilot to compute attitude at $50 \mathrm{~Hz}$. The autopilot is also connected to a blue-tooth modem which enables bi-directional communications with a ground based computer so that controller parameters and telemetry may be exchanged between the two systems. The autopilot decodes the servo position commands and generate the Pulse Width Modulation (PWM) signals to drive the servos.

For experimental purposes and safety, a mechanism is provided to allow the helicopter to be switched between manual and automatic control. A Hand Over Take Over (HOTO) system has been implemented on the helicopter allowing switching between these two modes. In automatic mode, the helicopter controls are set by the autopilot.
PC104 Based Flight Computer: The computational overhead required for real-time neural network based black-box system identification and subsequent use of the identified model for designing the adaptive controller of the Eagle helicopter necessitates more processing power than that provided by the MPC555. A PC104 based flight computer comprising a $650 \mathrm{MHz}$ Pentium III processor and $256 \mathrm{MB}$ of RAM, has therefore been implemented to do high level computation. Data is passed between the PC104 system and the autopilot using an RS-232 communication link (Figure 2).

A 2GB compact flash card on the PC104 allows the system to boot the RTLinux Real Time Operating System (RTOS) and the control software automatically on power up. The operating system includes X-Windows and a full set of development tools, so that a keyboard and monitor can be connected to the PC104 and the system used as a hardware in the loop development system. A convenient feature of the PC104 architecture is a native USB interface which has allowed a data logging feature using COTS memory stick devices. Flight data is recorded to the USB memory stick at $50 \mathrm{~Hz}$ and downloaded to a $\mathrm{PC}$ after each flight.

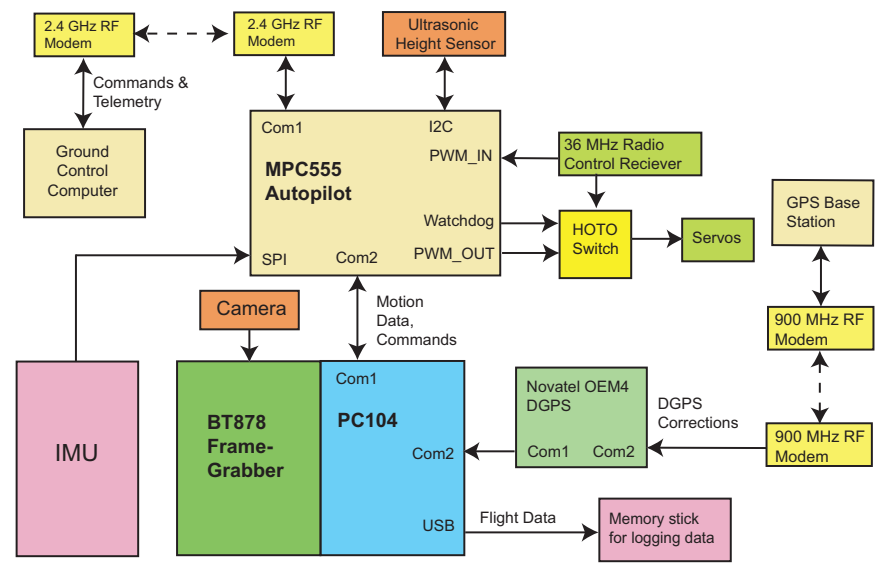

Fig. 2. PC104 and autopilot integration on Eagle platform

\subsection{Sensors:}

An IMU comprising three accelerometers and three $M u$ rata ENV-05D gyroscopes and three magnetometers is constructed to provide all the sensors needed for a complete Attitude Heading Reference System (AHRS). A 16bit Analog to Digital Converter (ADC) is installed in the IMU and interfaced to a PIC microcontroller which samples the sensors at $1600 \mathrm{~Hz}$ and communicates the data to the autopilot. The PC104 computer is interfaced to a Novatel OEM4-2GL differential GPS which provides $2 \mathrm{~cm}$ accuracy in position at a $20 \mathrm{~Hz}$ update rate using the real-time kinematic (RTK) mode and differential GPS corrections from a GPS base station. A PC104 frame grabber is interfaced to the PC104 to provide vision data from a small camera fitted on the nose of the helicopter.

\section{NEURAL NETWORK SYSTEM IDENTIFICATION}

In this section, the architecture and learning algorithm for the neural network based identification of a miniature Eagle helicopter is presented. 


\subsection{Formulation}

The Neural Network based black-box method is used to model the helicopter dynamics taking into account the interaction between all the inputs and outputs of the Multi-Input-Multi-Output (MIMO) system. The $m$ input, $p$ output discrete time representation of the $n^{\text {th }}$ order helicopter nonlinear dynamics is given by

$$
\begin{aligned}
\dot{x}(t+1) & =f[x(t), u(t)] \\
y(t) & =h[x(t)]
\end{aligned}
$$

where $x \in \Re^{n}$ is the state vector, $y \in \Re^{p}$ the output vector and $u \in \Re^{m}$ is the input vector at discrete time step $t$.

The input output relationship of a dynamical system can be described by AutoRegressive eXogenous inputs (ARX) model structure [Ljung (1987)]

$$
\begin{array}{r}
y(t)+a_{1} y(t-1)+\cdots+a_{n} y(t-n)= \\
b_{1} u(t-1)+\cdots+b_{m} u(t-m) .
\end{array}
$$

The parameter vector, $\theta$ is given by

$$
\theta=\left[\begin{array}{llll}
a_{1} & a_{2} \cdots a_{n} & b_{1} & b_{2} \cdots b_{m}
\end{array}\right]^{T} .
$$

The ARX model structure can be expressed as

$$
G(q, \theta)=\frac{B(q)}{A(q)}, \quad H(q, \theta)=\frac{1}{A(q)},
$$

where

$$
A(q)=1+a_{1} q^{-1}+\cdots+a_{n} q^{-n}
$$

and

$$
B(q)=b_{1} q^{-1}+\cdots+b_{m} q^{-m} .
$$

From the above model structure, the regressor vector can be defined as

$$
\psi(t)=[y(t-1), \cdots, y(t-n), \quad u(t-1), \cdots u(t-m)]^{T} .
$$

The number of past outputs and control inputs required to be fed for the system identification depends upon the order and the structure of the system. The number of regressors used for identification is decided by considering the sampling time and stabilization period of the dynamic system [ Lalot and Lecoeuche (2001)]. It is given by the relationship $\frac{t_{s t}}{t_{s}}$, where $t_{s t}$ is the stabilization period and $t_{s}$ is the sampling period of the system. The regressor vector should cover the entire stabilization period of the system, however this may not always be relied upon owing to the long stabilization period of the system. In the present study, the number of past inputs and outputs used were decided based on the dynamics by trial and error to suit the noisy data. This ARX model is used to identify the dynamics of Eagle helicopter by Feed Forward Neural Network (FFNN). The series parallel method suggested by Narendra and Parthasarathy (1990) and shown in Figure 3 is used for Neural Network Identification (NNID). The series parallel model is given by

$$
\begin{aligned}
\hat{y}(t)= & f\left[y_{p}(t-1), y_{p}(t-2), . ., y_{p}(t-n+1),\right. \\
& u(t), u(t-1), . ., u(t-m+1)],
\end{aligned}
$$

where $\hat{y}(t)$ is the predicted output of the network, $u(t)$ is the actual input to the plant, $y_{p}(t)$ is the true or measured output of the plant at time $t$.

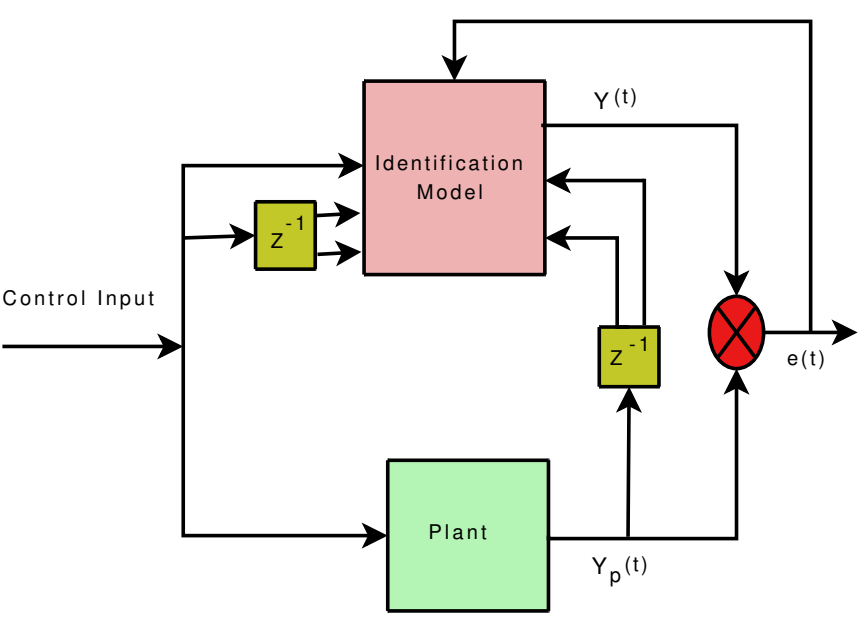

Fig. 3. Series-Parallel Model for NN Identification

\subsection{Neural Network Architecture and Training Algorithm}

Artificial Neural Networks (ANNs) are massively parallel distributed structures and have ability to learn through experience [Haykin (1994)]. Useful properties of ANNs such as nonlinearity, input/output mapping and adaptability have been exploited to model the dynamics of the Eagle helicopter. As one hidden layer with sufficient number of neurons is good enough to approximate any nonlinear function [Haykin (1994)], only one hidden layer is used for the present work. Levenberg-Marquardt (LM) optimization algorithm is used to minimize the cost function defined by the Mean Square Error (MSE) given by

$$
M S E=\frac{1}{N} \sum_{j=1}^{N}\left(d_{j}(n)-o_{j}(n)\right)^{2},
$$

where $d_{j}(n)$ is the desired response vector and $o_{j}(n)$ is the output vector of the $j^{\text {th }}$ element of the network output layer at iteration $n$ and $N$ is the total number of data points used for the training. The output of the network is computed by proceeding layer by layer through the network. The net internal activity level $V_{j}^{k}$ for a neuron $j$ in hidden layer $k$ is given by

$$
V_{j}^{k}(n)=\sum_{i=0}^{p} W_{j i}^{k}(n) Y_{i}^{k-1}(n)
$$

where $Y_{i}^{k-1}(n)$ is the output of neuron $i$ of the layer $k-1, W_{j i}^{k}(n)$ is the net weight of neuron $j$ in the layer $k$ connecting to the $i_{t h}$ neuron of layer $k-1$. The output of the neuron $j$ in layer $k$ is computed using the tanhyperbolic activation function given by

$$
Y_{j}^{k}(n)=1-\frac{2}{\exp \left(2 V_{j}^{k}(n)\right)+1}
$$

For NN training using LM algorithm, the gradient vector can be calculated as

$$
g(\theta)=J^{T}(\theta) e(\theta)
$$

where $J(\theta)$ is the system Jacobian matrix, $\theta$ is the parameter vector and $e(\theta)$ is the error vector defined by

$$
e(\theta)=\left[e_{1}, e_{2} \cdots e_{n}\right]^{T} .
$$

The minimum is found by iteratively solving the equation given by 


$$
\left(J(\theta)^{T} J(\theta)+\mu I\right) \Delta \theta=-J(\theta)^{T} e(\theta),
$$

where $\mu$ is a scalar quantity and $I$ is an identity matrix.

\section{RESULTS AND DISCUSSION}

In this section, the off-line and on-line identification of coupled and uncoupled dynamics of a miniature Eagle helicopter is presented.

\subsection{Flight Test Data Collection}

The Eagle helicopter instrumented to measure pitch rate $(q)$, pitch attitude $(\theta)$, roll rate $(p)$, roll attitude $(\phi)$, yaw rate $(r)$, longitudinal $\left(a_{x}\right)$, lateral $\left(a_{y}\right)$ and normal accelerations $\left(a_{z}\right)$ was test flown and various flight data was logged. The inputs to five servo actuators i.e. collective $\left(\delta_{c o l}\right)$, throttle, aileron $\left(\delta_{\text {lat }}\right)$, elevator $\left(\delta_{\text {lon }}\right)$ and tail rotor pitch $\left(\delta_{\text {ped }}\right)$ were also logged. A sampling frequency of $50 \mathrm{~Hz}$ was used for data collection.

\subsection{System Identification}

The number of regressors and network structure was decided by trial and error to obtain a best fit for input output data. For off-line modeling, as the high computational time can be afforded, the prediction accuracy decides the structure of the network and number of regressors. But this is not the case with on-line training as the training over the complete stack of data has to be finished within the sampling time which is $50 \mathrm{~Hz}$ for the present study. Therefore for on-line training, the structure of the system and the number of regressors has to be decided judiciously which would give minimum Root Mean Square Error (RMSE).

The dynamics of the UAV can be modeled either off-line or on-line depending upon the requirements. For off-line training, batch learning over all the patterns is performed to achieve the desired performance i.e. root mean square error (RMSE). In on-line training, one pattern is presented at each time step and the root mean square error corresponding to that pattern in that time step is minimized. The dynamics of the system may not be adequately represented by using one pattern for training at each time step. To improve the modeling accuracy, a batch or stack of data is presented to the network for training at each sampling time instead of only one pattern. The sliding window mode where a finite number of previous sampling periods is considered for minimizing the RMSE. The determination of the size of the window depends upon the prediction accuracy required and the processing time available. Large stack size will lead to better model approximation and transient performance at the cost of too much processing time but on the other hand smaller stack size will allow the training within the specified sampling time. For on board real time implementation of identification algorithm, $20 \mathrm{~ms}$ of processing time is available for each stack. Based on this, a stack size of 15 is used for on-line training in the present work.

The aerodynamics around the helicopter decides the basic characteristics of the helicopter dynamics. These change continuously with flight regime, thus on-line adaptation is necessary to model the dynamics of the system in real time.
The dynamics of a UAV is a six-degree-of-freedom coupled system. Under certain assumptions and conditions [Nelson (1997)], this six-degree-of-freedom system can be broken down into two 3 degree freedom dynamics. These are represented as the longitudinal dynamics and the lateral dynamics. The identification results are presented for both the off-line and on-line, uncoupled and coupled dynamics in the following subsections.

Off-line identification of longitudinal dynamics: For identification of decoupled longitudinal dynamics, the output vector $x$ and the input vector $u$ are considered as $x=\left[\theta, q, V_{x}\right]$ and $u=\left[\delta_{l o n}\right]$ respectively. Two past inputs and two past outputs are used in the regressor vector. The neural network has five neurons in the hidden layer. The predicted response from the NNID is plotted against the actual response of the helicopter in Figure 4. A statistical interpretation in terms of RMSE and variance is presented in Table 1. It can be seen from the Figure 4 and Table 1 that the NNID model captures the dynamics very well and the method is suitable for modeling the longitudinal dynamics of the Eagle helicopter.
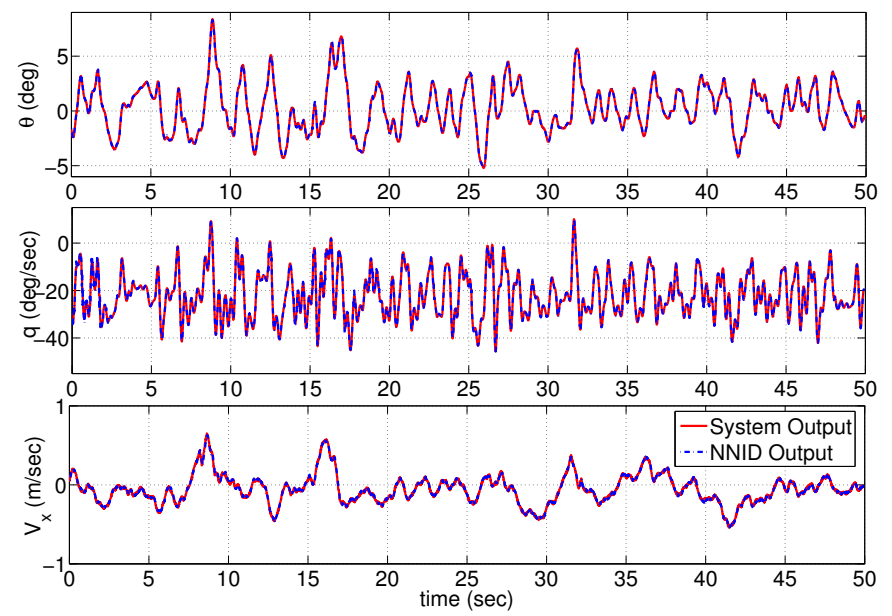

Fig. 4. Eagle Off-line NNID: Longitudinal Dynamics

Table 1. Off-line NNID Error Statistics: Longitudinal Dynamics

\begin{tabular}{|c|c|c|c|}
\hline Statistics & $\theta$ & $q$ & $V_{x}$ \\
\hline RMSE & 0.0051 & 0.0074 & 0.0007 \\
\hline variance & 0.0062 & 0.5851 & 0.0002 \\
\hline
\end{tabular}

Off-line identification of lateral dynamics: The output vector $x$ is represented by $x=\left[\phi, \psi, V_{y}\right]$ and the input vector by $u=\left[\delta_{l a t}, \delta_{\text {ped }}, \delta_{c o l}\right]$ for the lateral dynamics. Same number of hidden neurons, past inputs and past outputs as used for modeling the longitudinal dynamics are employed for network training. The predicted responses from the NNID model are plotted against the actual responses of the helicopter in Figure 5 for comparison. The statistical results for neural network model response is presented in Table 2. Figure 5 and Table 2 suggests that the NNID model is suitable for system identification with acceptable error characteristics. 


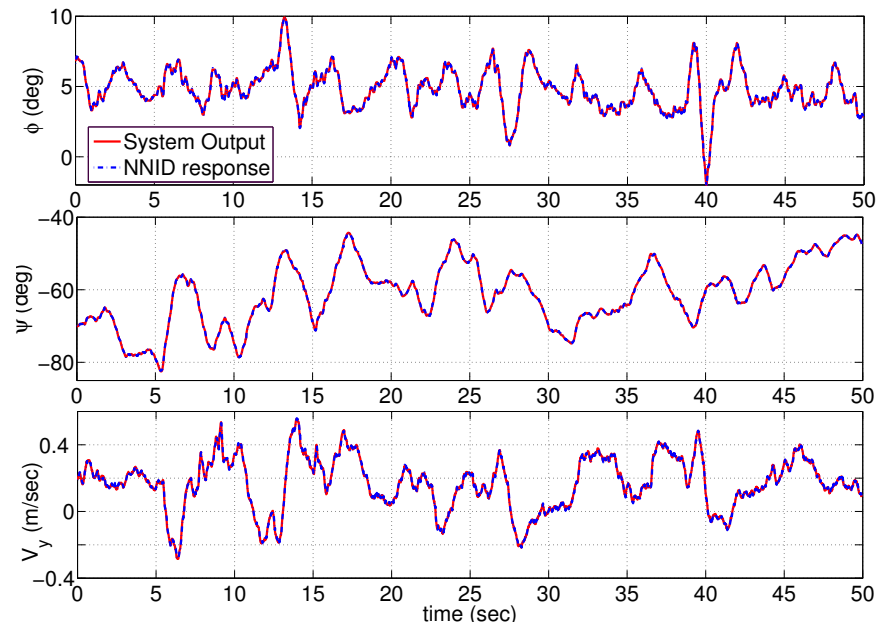

Fig. 5. Eagle Off-line NNID: Lateral Dynamics

Table 2. Off-line NNID Error Statistics: Lateral Dynamics

\begin{tabular}{|c|c|c|c|}
\hline Statistics & $\phi$ & $\psi$ & $V_{y}$ \\
\hline RMSE & 0.0318 & 0.2137 & 0.004 \\
\hline variance & 0.0063 & 0.0208 & 0.0001 \\
\hline
\end{tabular}

Off-line identification of coupled six-degree-of-freedom dynamics: Most of the times, it may not be possible to decouple the six-degree-of-freedom system into two threedegree-of-freedom systems. Therefore the dynamics of the helicopter need to be modeled as a coupled six-degreeof-freedom system. For identification of the coupled sixdegree-of-freedom dynamics of the Eagle helicopter, the state vector is represented by $x=\left[\phi, \theta, \psi, V_{x} V_{y}, V_{z}\right]$ and the input vector by $u=\left[\delta_{\text {long }}, \delta_{\text {lat }}, \delta_{\text {ped }}, \delta_{\text {col }}\right]$. Twelve neurons in the hidden layer and two past inputs and two past outputs were used for neural network training. The identification results for attitudes and velocities are plotted against the actual responses of the helicopter in Figures 6 and 7 respectively. The error statistics of the predicted responses are presented in Table 3 . The results indicate that the neural network is capable of imitating the dynamics of the helicopter.

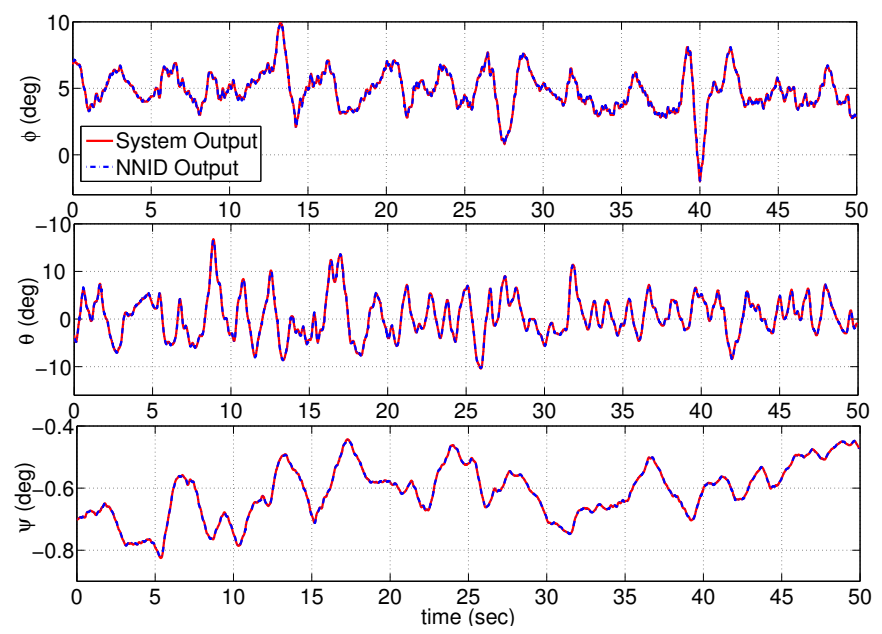

Fig. 6. Eagle Off-line NNID: Coupled Dynamics

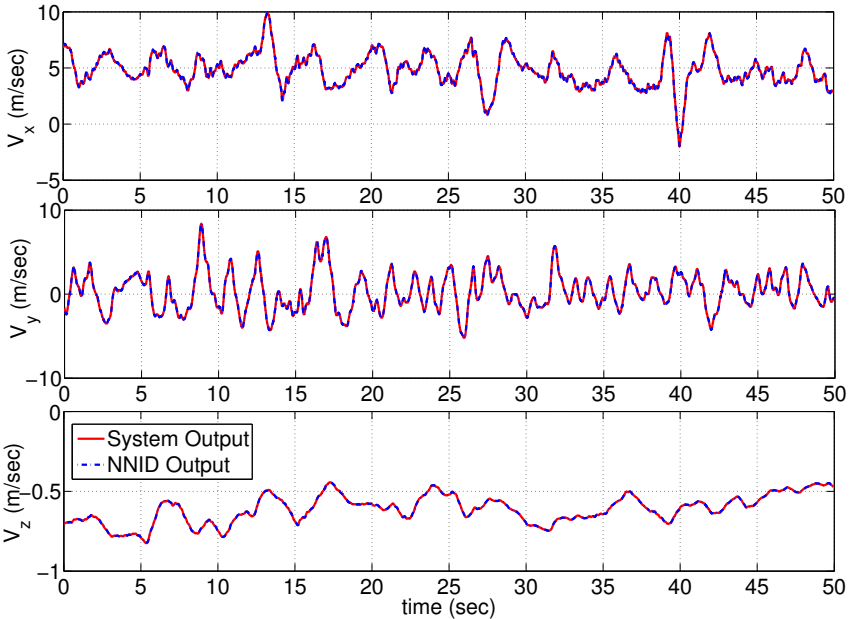

Fig. 7. Eagle Off-line NNID: Coupled Dynamics

Table 3. Off-line NNID Error Statistics: Coupled Dynamics

\begin{tabular}{|c|c|c|}
\hline Statistics & RMSE & Variance \\
\hline$\phi$ & 0.0210 & 0.0059 \\
\hline$\theta$ & 0.1237 & 1.0451 \\
\hline$\psi$ & 0.0002 & 0.0000 \\
\hline$V_{x}$ & 0.0196 & 0.0002 \\
\hline$V_{y}$ & 0.0019 & 0.0001 \\
\hline$V_{z}$ & 0.0056 & 0.0002 \\
\hline
\end{tabular}

On-line identification of uncoupled longitudinal dynamics: For the on-line modeling of helicopter longitudinal dynamics, five hidden neurons were used in the hidden layer. The predicted and actual responses of the helicopter are plotted in Figure 8. The error statistics is provided in Table 4. Due to the limited availability of processing time, accuracy has been compromise as can be seen from the results.

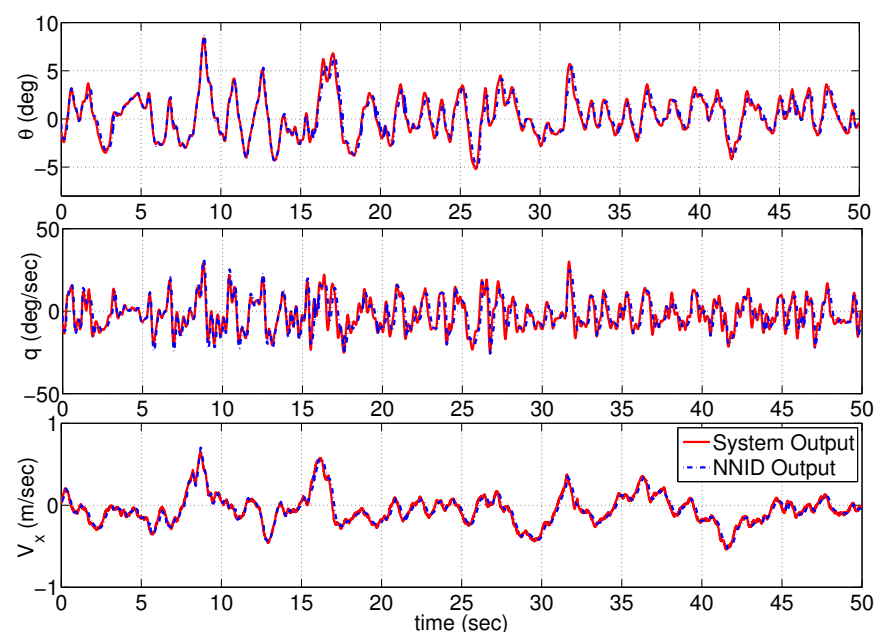

Fig. 8. Eagle On-line NNID: Longitudinal Dynamics

Table 4. On-line NNID Error Statistics: Longitudinal Dynamics

\begin{tabular}{|c|c|c|c|}
\hline Statistics & $\theta$ & $q$ & $V_{x}$ \\
\hline RMSE & 0.4292 & 0.700 & 0.0156 \\
\hline variance & 0.3935 & 0.1010 & 0.0018 \\
\hline
\end{tabular}


On-line identification of uncoupled lateral dynamics: Five neurons in the hidden layer are used for on-line training of the lateral dynamics as well. The responses and error statistics are presented in Figure 9 and Table 5 respectively. The deterioration in the results due to online training is clearly visible. From the adaptive controller design prospective, these results are acceptable.
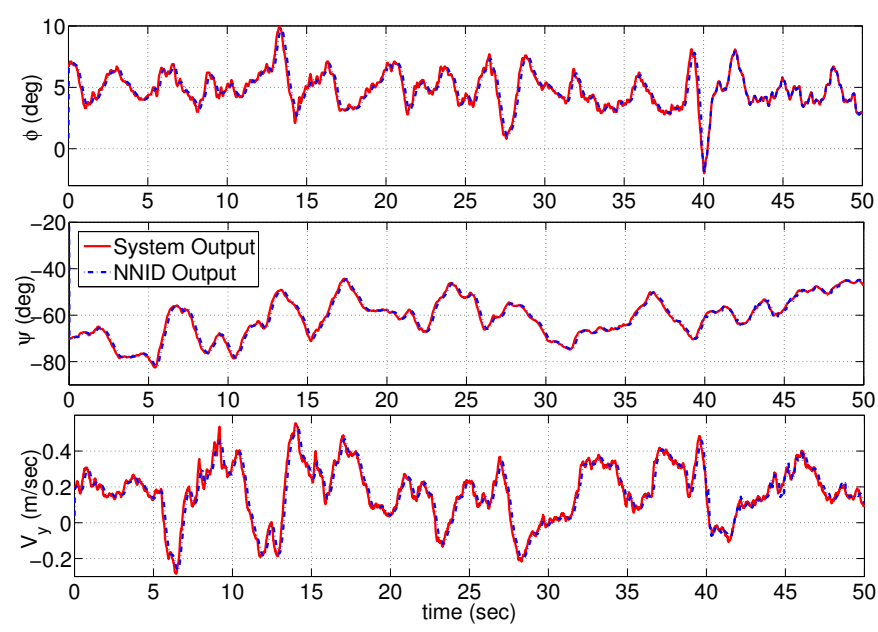

Fig. 9. Eagle On-line NNID: Lateral Dynamics

Table 5. On-line NNID Error Statistics: Lateral Dynamics

\begin{tabular}{|c|c|c|c|}
\hline Statistics & $\phi$ & $\psi$ & $V_{y}$ \\
\hline RMSE & 0.5205 & 0.5504 & 0.0107 \\
\hline variance & 0.0208 & 0.3122 & 0.0016 \\
\hline
\end{tabular}

\section{CONCLUSION}

In the present study, the nonlinear dynamics of the Eagle helicopter is identified from the flight data. Neural network based black-box approach is used to model the dynamics both off-line and on-line. The predicted responses from these neural network models are compared with the actual responses of the Eagle helicopter. The off-line model seems to perform better as compared to the on-line model. This can be attributed to the additional training time and bigger batch size available for off-line training. Currently the research is directed towards the design of suitable controllers based upon these neural network models.

\section{REFERENCES}

Ramazan Tasaltin Aydogan Savran and Yasar Becerikli. Intelligent adaptive nonlinear flight control for a high performance aircraft with neural networks. ISA Transactions, 45(2):225-247, April 2006.

Girish Chowdhary and Ravindra Jategaonkar. Aerodynamic parameter estimation from flight data applying extended and unscented kalman filter. AIAA Atmospheric Flight Mechanics Conference, August 2006.

A. K. Ghosh, S.C. Raisinghani, and S. Khubchandani. Estimation of aircraft lateral-directional parameters using neural networks. Journal of Airraft, 35(6):876-881, 1998.

Simon Haykin. Neural Networks: A Comprehensive Foundation. Macmillan College Publishing Company, 1994.

Ravindra Jategaonkar, Dietrich Fischenberg, and Wolfgang von Gruenhagen. Aerodynamic modeling and system identification from flight data-recent applications at dlr. Journal of Aircraft, 41(4):681-691, july-august 2004.

Abhijit Kallapur, Shaaban Ali, and Sreenatha Anavatti. Experiences using emid and ekf for uav online identification. In Third International Conference on Autonomous Robots and Agents (ICARA-2006), pages 207-212, Palmerston North, New Zealand, December 2006. Springer Verlag.

M. Vijaya Kumar, S. N. Omkar, Ranjan Ganguli, Prasad Sampath, and S. Suresh. Identification of helicopter dynamics using recurrent neural networks and flight data. Journal Of the American Helicopter Society, pages 164-174, April 2006.

Sylvain Lalot and Stephane Lecoeuche. Online identification of heat dissipaters using artificial neural networks. Journal of Mechanical Engineering, pages 411416, 2001.

Lennart Ljung. System Identification: Theory for the user. Prentice-Hall, Inc., Englewood Cliffs, New Jersey, 1987.

Bernard Mettler, Mark B. Tischler, and Takeo Kanade. System identification modelling of a small-scale unmanned rotorcraft for flight control design. Journal of the American Helicopter Society, pages 50-63, January 2002.

Kampati S. Narendra and Kannan Parthasarathy. Identification and control of dynamic systems using neural network. IEEE Transactions on Neural Networks, 1(1): 4-27, march 1990.

Robert C. Nelson. Flight Stability and Automatic Control. McGraw-Hill, 1997.

S. C. Raisinghani and A. K. Ghosh. Two new techniques for aircraft parameter estimation using neural networks. Aeronautical journal, pages 25-29, 1998.

J. R. Raol. Neural network based parameter estimation of unstable aerospace dynamic systems. IEE Proceeding in Control Theory Application, 141(6):385-388, Nov 1994. 